\title{
A refocus on the advances of single-cell biomedicine
}

\author{
William Wang • Xiangdong Wang
}

Received: 20 July 2020 / Accepted: 31 July 2020/Published online: 10 August2020

(C) Springer Nature B.V. 2020

Single-cell RNA-sequencing (scRNA-seq) is an important technology to provide new insights into molecular mechanisms of intra- and intercellular regulations in special cells, defining new categories of cell types and developing and validating new clusters of biomarkers and targets. We are currently focused on scRNA-seq and its role in unknown intracellular communications and how it interacts among transcriptional networks and signal pathways in single-cell biology, biomedicine, and toxicology. Cell Biology and Toxicology published its first paper related to "single cell" in 1988 to address the development of thioguanine-resistant primary clones from single-cell suspensions isolated from dog and human kidneys (Turker et al. 1988). The first study on single-cell toxicology was reported in 2000 by measuring the extent of DNA damage induced by herbicide in alkaline single-cell microgel electrophoresis (Villarini et al. 2000). From 2017, the journal started to pay special attention to the values of single-cell measurements to develop a better understanding of transcriptomic profiles in cell biology using scRNA-seq, the genetic development and molecular mechanisms of intra-clonal and inter-clonal heterogeneity in cancer using scDNA-seq, and the dynamic phenotypes and responses of a cell to drugs by integrating it with morphological phenomes

William Wang and Xiangdong Wang authors contribute to this article equally as the first authors

W. Wang $\cdot X$. Wang $(\bowtie)$

Zhongshan Hospital Institute of Clinical Science, Fudan University Shanghai Medical College, Shanghai, China e-mail: xdwang@fuccb.com and CRISPR screening (Chu et al. 2017; Wang and Wang 2017; Wang et al. 2017). Single-cell RNA and DNA sequencing was a key approach in uncovering the heterogeneity and molecular regulations in resistant cells and circulating tumor cells, based on which a model of the artificial intelligent single cell can be established for clinical application. This model could help to achieve rapid bioinformatics analyses of multiple dimensional omics data, fast indications/suggestions in decisionmaking, and efficient therapies for complex severe diseases (Wang et al. 2018; Zeng et al. 2018; Zhu et al. 2018; Yin et al. 2019).

An in-depth understanding of pre-, inter-, and posttranscriptional profiles at a cell has heralded a new era of single-cell biomedicine. Single-cell nuclear elements are major parts of pre-transcriptional profiles, and they are responsible for regulating the interaction between transcriptional factors, DNA elements, and genome organizations and for controlling the developmental evolution and dynamic formation of genotoxicity (Zeng et al. 2020). Single-cell gene editing (e.g., CRISPR-Cas9, Cas9, and Cas13) can be a new strategy for defining target-dependent molecular networks and mechanism of cell biology and toxicology (Wang et al. 2019; Yan et al. 2019). For example, the regulation of rs2076295 in desmoplakin expression and dysfunction responsible for idiopathic pulmonary fibrosis was investigated in human bronchial epithelial cells using CRISPR/Cas9 editing (e.g., regional deletion, indel, CRISPRi, and single-base editing) with scRNA-seq (Hao et al. 2020). Target-oriented regulations and associated networks demonstrated cell type-specific expressions of target 
genes and its regulated genes in a clear gene-controlled cell. Post-transcriptional profiles of the single cells are also a critical part in single-cell biomedicine in cell biology and toxicology (Qiao and Wang 2019; Gil et al. 2019; Zheng et al. 2020). It is important to define the relative abundances of protein products, posttranslational modifications, protein localization, turnover, protein interactions, and protein function at a single-cell level to design proteomic profiles of body fluids and tissues that can identify biomarkers and therapeutic targets. Gene editing can be an alternative for new therapies with the specificity and efficiency of ontarget sites. On the other hand, off-targets of various genome editing should be emphasized for clinical application (Wang et al. 2019; Wang and Wang 2019; Zheng et al. 2020).

In addition to the cell type-specific expressions of target genes, interactions, and regulations among targetdependent network elements, scRNA-seq is a powerful new approach to evaluate precision toxicology at a target cell by combining multidimensional omics and pathway analysis. Zhang et al. (2020) studied molecular mechanisms of aflatoxin B1-induced hepatotoxicity using scRNA-seq and discovered that the single cell reduced representation of bisulfite sequencing and defined the transcriptomic profiles of targeted cells associated with dysfunctions (e.g., mitochondrial membrane potential, reactive oxygen species, and DNA methylation). Recently, scRNA-seq has been widely applied for detecting cell toxicity and response to side effects, adverse effects, and serious effects of new drugs in clinical trials and investigations. The strategy of combining scRNA-seq with validation was used to evaluate drug and environmental toxicity in embryonic organ system development, organ organoid differentiation monitoring, toxicity testing, and defining target-specific and/or new differentiated cell populations to face new challenges.

The combination of scRNA-seq and biotechnologies is developing rapidly to address more complex systemic mechanisms of targets. He et al. (2020) combined a deep learning algorithm with spatial transcriptomics and histology images to capture high-resolution gene expression heterogeneity in breast cancer. This important approach has great potential for clinical translation to rapidly analyze spatial and local alternations in targetassociated transcriptional profiles and the expressions of genes and proteins and to increase the quality and level of clinical molecular pathology. If spatial transcriptomics can be integrated with location transcripts for scRNA-seq, higher sequencing depths can be achieved that can capture detailed and larger quantities of genes while complimenting the loss of spatial information when single cells are isolated from the tissue. Ren et al. (2020) developed a new approach named Cellular Spatial Organization mapper (CSOmap) that aims to reconstruct the spatial organization of the tissue using scRNA-seq information. Validated CSOmap can then be used to reconstruct spatial cellular organizations in multiple organs of human and mouse. This is an exciting development in single-cell biomedicine from scRNA-seq that can simulate cell spatial organization and interaction in the tissue/organ and a potential tool for clinical application to describe the perturbation of genes or cell types in the progression of disease. For example, CSOmap has been applied in reconstructing the exocrine and endocrine compartments of the pancreas from recapitulated ligand receptors, detecting the difference of spatial characteristics between human normal and fibrotic alveoli and moding the situation of immune cell interaction and intercommunication within liver cancer, which is consistent with immunohistochemical imaging of the tissue sample (Ren et al. 2020).

scRNA-seq can describe more molecular mechanisms and have more applicable values than transcriptomic profiles. Qian et al. (2020) used scRNA-seq with protein information to build up a pancancer blueprint of stromal cell heterogeneity, character 68 stromal cell populations based on phenotypes of genes, transcription factors, metabolic activities, and tissue-specific expression and to describe the similarity of intercellular and intertype heterogeneities among cancer-associated tissues/organs. This is an example on how to develop a more comprehensive and multidimensional understanding of each subpopulation and the characters associated and specialized with cancer types, locations, and therapies. According to canonical correlation analysis, about $70 \%$ stromal cell populations have cancer phenotypes, e.g., 5 clusters of endothelial cells shared between cancer types, fibroblasts with the highest cancer type specificity, and dendritic cells with low cancer types (Qian et al. 2020). Roider et al. (2020) characterized the cell composition of nodal B cell lymphomas and lymph node-derived $\mathrm{T}$ cell populations as well as gene expression signatures driving $\mathrm{B}$ cell heterogeneity using scRNA-seq and transcriptional intratumor heterogeneity using multicolor flow cytometry. 
This is an outstanding example of how scRNA-seq combined with transcriptome-informed flow cytometry can investigate the intercellular communication and interaction between $T$ cells and malignant $B$ cells within the lymph node microenvironment and the dynamic and aggressive components of the distinct transcriptional, genomic, and drug response profiles. Transcriptionally distinct malignant subpopulations and subpopulationspecific drug sensitivity were uncovered for the development of precision medicine. These scRNA-seq-extended combinations provide a new avenue for verifying molecular mechanisms from single-cell transcriptomes and intercellular communications to large subpopulations of target cells' shared characteristics.

Applications of scRNA-seq and its extensions are rapidly developing and applied in new fields, e.g., to identify the ACE2 receptor in target cells during COVID-19 infections, establishing public servers pooled with scRNA-seq data for deep mining of intercommunication, developing scRNA-seq-based targetscreening system integrated with CRISPR, and creating an intelligent single-cell mode. The terminal goal is to apply scRNA-seq and analytic methods with other approaches for identifying target-, therapy-, disease-, and drug response-specific subpopulations and developing new therapies on the basis of single-cell biomedicine. The major obstacles to reach this goal are high costeffectiveness, variety in clinical sample handling, lack of efficient analyses, a slow process, and varying understanding of scRNA-seq among experts. scRNA-seqbased combinations with multi-omics and trans-omics can provide new insights in understanding molecular mechanisms, intercellular communication, and the specific interactions of disease depending on its type, stage, severity, and response to drugs. We hope that further improvement and development in single-cell biomedicine can help to identify new biomarkers and therapies based on single cells.

In conclusion, it has taken decades to realize the importance of the advances in single-cell biomedicine as a new emerging discipline in Cell Biology and Toxicology. scRNA-seq plays critical roles in the understanding of transcriptomic profiles, cell-cell communications, and intracellular signal network regulations. scRNA-seq is also applied for uncovering biology-based and disease-specific biomarkers and therapeutic targets and for monitoring cell sensitivity and responses to drugs. Here, we specially emphasize the importance that scRNA-seq-combined new approaches provide multidimensional insights in the interactions among genes, proteins, and transcriptional factors, reconstruction of cell spatial organization from isolated single cells to cells within tissue, prediction of cells subpopulations affected by target cells within tissue/organs, and the assembly of an artificial intelligent cell.

\section{References}

Chu MP, Kriangkum J, Venner CP, Sandhu I, Hewitt J, Belch AR, et al. Addressing heterogeneity of individual blood cancers: the need for single cell analysis. Cell Biol Toxicol. 2017;33(2):83-97. https://doi.org/10.1007/s10565-0169367-4.

Gil J, Betancourt LH, Pla I, Sanchez A, Appelqvist R, Miliotis T, et al. Clinical protein science in translational medicine targeting malignant melanoma. Cell Biol Toxicol. 2019;35(4):293-332. https://doi.org/10.1007/s10565-01909468-6.

Hao Y, Bates S, Mou H, Yun JH, Pham B, Liu J, et al. GWAS functional variant rs2076295 regulates desmoplakin (DSP) expression in airway epithelial cells. Am J Respir Crit Care Med. 2020. https://doi.org/10.1164/rccm.201910-1958OC.

He B, Bergenstråhle L, Stenbeck L, Abid A, Andersson A, Borg $\AA$, et al. Integrating spatial gene expression and breast tumour morphology via deep learning. Nat Biomed Eng. 2020. https://doi.org/10.1038/s41551-020-0578-x.

Qian J, Olbrecht S, Boeckx B, Vos H, Laoui D, Etlioglu E, et al. A pan-cancer blueprint of the heterogeneous tumor microenvironment revealed by single-cell profiling. Cell Res. 2020. https://doi.org/10.1038/s41422-020-0355-0.

Qiao T, Wang X. A new light of proteomics in cell biology and toxicology. Cell Biol Toxicol. 2019;35(4):289-91. https://doi.org/10.1007/s10565-019-09492-6.

Ren X, Zhong G, Zhang Q, Zhang L, Sun Y, Zhang Z. Reconstruction of cell spatial organization from single-cell RNA sequencing data based on ligand-receptor mediated self-assembly. Cell Res. 2020. https://doi.org/10.1038 /s41422-020-0353-2.

Roider T, Seufert J, Uvarovskii A, Frauhammer F, Bordas M, Abedpour N, et al. Dissecting intratumour heterogeneity of nodal B-cell lymphomas at the transcriptional, genetic and drug-response levels. Nat Cell Biol. 2020;22(7):896-906. https://doi.org/10.1038/s41556-020-0532-x.

Turker MS, Monnat RJ Jr, Fukuchi K, Johnston PA, Ogburn CE, Weller RE, et al. A novel class of unstable 6-thioguanineresistant cells from dog and human kidneys. Cell Biol Toxicol. 1988;4(2):211-23. https://doi.org/10.1007 /BF00119247.

Villarini M, Scassellati-Sforzolini G, Moretti M, Pasquini R. In vitro genotoxicity of terbutryn evaluated by the alkaline single-cell microgel-electrophoresis "comet" assay. Cell Biol Toxicol. 2000;16(5):285-92. https://doi.org/10.1023 /a:1026794213308.

Wang W, Wang X. Single-cell CRISPR screening in drug resistance. Cell Biol Toxicol. 2017;33(3):207-10. https://doi. org/10.1007/s10565-017-9396-7. 
Wang DC, Wang X. Off-target genome editing: a new discipline of gene science and a new class of medicine. Cell Biol Toxicol. 2019;35(3):179-83. https://doi.org/10.1007 /s10565-019-09475-7.

Wang W, Zhu B, Wang X. Dynamic phenotypes: illustrating a single-cell odyssey. Cell Biol Toxicol. 2017;33(5):423-7. https://doi.org/10.1007/s10565-017-9400-2.

Wang W, Gao D, Wang X. Can single-cell RNA sequencing crack the mystery of cells? Cell Biol Toxicol. 2018;34(1):1-6. https://doi.org/10.1007/s10565-017-9404-y.

Wang W, Hou J, Zheng N, Wang X, Zhang J. Keeping our eyes on CRISPR: the "Atlas" of gene editing. Cell Biol Toxicol. 2019;35(4):285-8. https://doi.org/10.1007/s10565-01909480-w.

Yan F, Wang W, Zhang J. CRISPR-Cas12 and Cas13: the lesser known siblings of CRISPR-Cas9. Cell Biol Toxicol. 2019;35(6):489-92. https://doi.org/10.1007/s10565-01909489-1.

Yin J, Wang Z, Li G, Lin F, Shao K, Cao B, et al. Characterization of circulating tumor cells in breast cancer patients by spiral microfluidics. Cell Biol Toxicol. 2019;35(1):59-66. https://doi.org/10.1007/s10565-018-09454-4.

Zeng Y, Chen X, Gao H, Wang X. An artificial intelligent single cell is part of the cell dream world. Cell Biol Toxicol.
2018;34(4):247-9. https://doi.org/10.1007/s10565-0189433-1.

Zeng Y, Wang X, Zhang J. Single-cell biomedicine: roles of single-cell nuclear elements. Cell Biol Toxicol. 2020;36(1): 1-3. https://doi.org/10.1007/s10565-020-09515-7.

Zhang B, Dai Y, Zhu L, He X, Huang K, Xu W. Single-cell sequencing reveals novel mechanisms of Aflatoxin B1induced hepatotoxicity in S phase-arrested L02 cells. Cell Biol Toxicol. 2020. https://doi.org/10.1007/s10565-02009547-z.

Zheng N, Li L, Wang X. Molecular mechanisms, off-target activities, and clinical potentials of genome editing systems. Clin Transl Med. 2020;10(1):412-26. https://doi.org/10.1002 /ctm2.34.

Zhu Z, Qiu S, Shao K, Hou Y. Progress and challenges of sequencing and analyzing circulating tumor cells. Cell Biol Toxicol. 2018;34(5):405-15. https://doi.org/10.1007 /s10565-017-9418-5.

Publisher's note Springer Nature remains neutral with regard to jurisdictional claims in published maps and institutional affiliations. 